\title{
Wem gehören die großen Unternehmen? Restrukturierung des Eigentums während der Finanzkrise in Deutschland und den USA
}

Eigentum an großen Unternehmen konstituiert gesellschaftliche Macht. Der Beitrag stellt die Frage: Wem gehören die 200 größten realwirtschaftlichen Unternehmen in Deutschland und in den USA? Hat die Finanzkrise die Eigentümerstrukturen verändert? Die Befunde zeigen, dass in beiden Ländern Finanzinvestoren wie BlackRock einen erheblichen Anteil an den Aktien großer Nicht-Finanzunternehmen besitzen und die Konzentration des Aktienbesitzes während der Krise zugenommen hat.

WERNER NIENHÜSER, DAVID PEETZ, GEORGINA MURRAY

\section{Fragestellung und Vorgehensweise}

Die Frage, wer die großen Unternehmen über Eigentumsrechte kontrolliert, wird in den Sozial- und Wirtschaftswissenschaften aus gutem Grund immer wieder gestellt (vgl. z. B. Fligstein/Brantley 1992; Streeck/Höpner 2003; Windolf/Nollert 2001; Vitali et al. 2011). Die Eigentümer großer Unternehmen verfügen in einer Gesellschaft über erhebliche Macht, und ihr Handeln beeinflusst das Leben vieler Menschen. Unsere erste Frage richtet sich also darauf, wer das Eigentum an den großen Unternehmen (den Top-200) in Deutschland und in den USA hält und ob sich Veränderungen in der Eigentümerstruktur nach der 2008/2009 ausgebrochenen Finanzkrise zeigen. Dabei betrachten wir nur das Eigentum an den realwirtschaftlichen, Güter und Dienstleistungen produzierenden Unternehmen. Finanzinvestoren und ähnliche Finanzunternehmen beziehen wir als Eigentümer mit ein. Wir werden u. a. sehen, dass in beiden Ländern Finanzinvestoren bzw. Vermögensverwalter, wie das in New York ansässige Unternehmen BlackRock, einen erheblichen Anteil der Aktien des produktiven Kerns der Volkswirtschaften besitzen.

Zweitens stellt sich die Frage, ob sich die Strategien der Anleger verändert haben. Unsere Befunde zeigen u. a., dass die großen Aktienbesitzer (Top-30) in Deutschland ihre Anlagen - anders als in den USA - auf nur wenige Unternehmen konzentrieren, während in den USA stärker eine
Strategie vorzufinden ist, bei der die Anleger versuchen, in sehr vielen Unternehmen der größte oder zweitgrößte Aktieninhaber zu sein.

Unsere dritte Frage: Hat sich die Konzentration des Aktienbesitzes durch die letzte Finanzkrise 2008/2009 verändert? Wir vermuten, dass die Eigentumskonzentration durch den krisenbedingt verschärften Wettbewerb zugenommen hat. Um auch hier einen Befund knapp vorwegnehmend zu skizzieren: In den beiden betrachteten Ländern hat die Konzentration des Aktienbesitzes zugenommen, in Deutschland allerdings auf einem deutlich geringeren Niveau.

Viertens wollen wir wissen, ob es eine stärkere Finanzialisierung, d. h. eine Verstärkung der Bedeutung von Finanzmärkten, -akteuren und -institutionen, gegeben hat. ${ }^{1}$ Hat der Anteil des Eigentums zugenommen, den Finanzunternehmen in anderen Unternehmen kontrollieren, und hat es Verschiebungen des Aktienbesitzes innerhalb des Finanzkapitals, insb. von Banken hin zu Finanzunternehmen (z. B. Investmentfonds und Vermögensverwaltungen), gegeben? Anders als wir es erwartet hatten, können wir hier bezogen auf das Eigentum an Nicht-Finanzunternehmen nur einen schwachen Finanzialisierungsschub beobachten. Allerdings betrachten wir nur einen kurzen Zeitraum.

1 Zum Begriff der Finanzialisierung vgl. Heires/Nölke (2011, 2014); Lapavitsas (2011); van Treeck (2009); van der Zwan (2014). 
Für die Analyse verwenden wir als Ausgangspunkt die Daten über die 200 jeweils umsatzstärksten Nicht-Finanzunternehmen in den USA und in Deutschland sowie über die Anleger, die die Aktien in diesen Unternehmen halten (mehr zum Datensatz in Abschnitt 2.2). ${ }^{2}$ Deutschland und die USA haben wir zum einen ausgewählt, weil diese beiden Länder massiv, wenngleich in unterschiedlichem Maße, von der Finanzkrise getroffen wurden. ${ }^{3}$ Ein zweiter Grund für die Länderauswahl: Die in allen vier oben skizzierten Fragen angesprochenen Veränderungen finden vor dem Hintergrund des jeweils länderspezifischen Institutionengeflechts statt und werden von diesem beeinflusst, gebrochen oder verstärkt. Die beiden Länder repräsentieren unterschiedliche Formen des Kapitalismus. Die USA können als der typische Fall einer liberalen Marktwirtschaft gelten, Deutschland entspricht einer koordinierten Marktwirtschaft (Hall/Soskice 2001).

Mit unseren Analysen wollen wir einen Beitrag zur empirischen Beantwortung der oben skizzierten Fragen leisten und zugleich etwas zur Theoriedebatte beisteuern. Unsere Befunde zeigen, dass sich innerhalb unterschiedlicher Kapitalismen Veränderungen auf der Mikroebene zeigen, die in unserem Fall auf der Unternehmensebene stattfinden; diese Veränderungen spielen sich zunächst innerhalb des institutionellen Rahmens ab, können diesen aber so stark unter Druck setzen, dass sich auch eine Veränderung der regulativen Institutionen auf der Meso- oder Makroebene ergeben kann. Damit würde sich langfristig auch der institutionelle Rahmen für die Unternehmen wandeln.

Wir gehen wie folgt vor: Wir skizzieren zunächst in Abschnitt 2 unseren theoretisch-konzeptionellen Hintergrund und entwickeln auf dieser Basis Hypothesen. Darüber hinaus erläutern wir die für die Analyse verwendete Datenbasis. In Abschnitt 3 nehmen wir eine namentliche Analyse der wichtigsten Anteilseigner vor. Abschnitt 4 widmet sich der Frage nach den Strategien der Anleger. Die möglichen Veränderungen in der Konzentration des Aktienbesitzes sind Gegenstand von Abschnitt 5. Danach (6) geht es um die Frage, ob nach der Krise eine stärkere Finanzialisierung als vor der Krise zu verzeichnen ist. Zum Schluss des Beitrages diskutieren wir, welche Folgen sich aus den Veränderungen der Eigentümerstrukturen für die Unternehmensführung und Realisierung der Arbeitnehmerinteressen ergeben können (7).

\section{Theoretisch-konzeptioneller Hintergrund und Datenbasis}

\subsection{Theoretisch-konzeptioneller Hintergrund}

Um landesbedingte Unterschiede der Krisenwirkungen besser zu verstehen, greifen wir auf den „Varieties of Capitalism
(VoC)“-Ansatz von Hall und Soskice (2001) zurück und ergänzen diesen um krisentheoretische Überlegungen.

Der VoC-Ansatz unterscheidet zwischen liberalen und koordinierten Marktwirtschaften. Die USA und auch das Vereinigte Königreich entsprechen dem ersten, Deutschland und die skandinavischen Ländern dem zweiten Typ. Zentral ist die Annahme, dass beide Formen des Kapitalismus eine jeweils in sich stimmige Konfiguration von Institutionen bilden, innerhalb derer Unternehmen agieren (und die Struktur der Konfiguration reproduzieren): Unternehmen sind eingebettet in ein Finanzierungs-, Bildungs-, Arbeitsbeziehungs- und Rechtssystem.

In liberalen Marktwirtschaften findet die Koordination zwischen Unternehmen über Märkte und Preise statt. Die Finanzierung von Unternehmen erfolgt weniger über Banken, sondern über Kapitalmärkte, die durch eine relativ hohe Transparenz und verstreuten Aktienbesitz gekennzeichnet sind. In koordinierten Marktwirtschaften ist der Wettbewerb als Koordinationsmechanismus schwächer. Es wird stärker koordiniert über den Mechanismus „Organisation“, d. h. über Kommunikation, etwa mit Hilfe von Unternehmensverbänden und des Staates. Ein weiterer Unterschied besteht darin, dass sich die Unternehmen in koordinierten Marktwirtschaften stärker über Banken und weniger direkt über anonyme Kapitalmärkte finanzieren; sie sind damit weniger stark der Konkurrenz um Finanzierung ausgesetzt und ihre Profiterwartungen dürften langfristiger ausgerichtet sein.

Der VoC-Ansatz wird in der Literatur nach wie vor virulent diskutiert (siehe zu dieser umfangreichen Diskussion stellvertretend Schneider/Paunescu 2012). Kritisiert wird unter anderem seine (zu) einfache Dichotomisierung von polit-ökonomischen Systemen. Darüber hinaus fehle es ihm an einer theoretischen Basis (Heyes et al. 2012). Beide Punkte treffen zu. Gleichwohl halten wir an der Dichotomisierung fest, da wir lediglich zwei Länder miteinander vergleichen, deren Einordnung an den Endpunkten eines Kontinuums (zwischen liberalen und koordinierten Marktwirtschaften) relativ unstrittig ist. Wesentlich ist uns folgender Punkt: Wir untersuchen Veränderungen innerhalb der jeweiligen Form des Kapitalismus, das heißt, Veränderungen auf der Ebene der Gesamtheit (bzw. einer wichtigen Teilgesamtheit) der Unternehmen und weniger solche auf der regulierungs- oder institutionsbezogenen Ebene. Der VoC-Ansatz liefert uns Anhaltspunkte für die Unterscheidung des jeweiligen Länderkontextes, innerhalb dessen die Unternehmen agieren. Hier schließt sich die

2 Wir danken der Griffith-Universität für die Finanzierung des Datenzugangs und der Fakultät der Universität Duisburg-Essen für die teilweise finanzielle Förderung des Forschungsaustausches durch Reisemittel.

3 Vgl. zu einem vier Länder erfassenden, Deutschland allerdings wenig behandelten Vergleich: Peetz et al. (2013). 
bereits aufgeworfene Frage an, ob nicht zumindest mittelfristig Veränderungen auf der Ebene der Unternehmen die institutionellen Regulierungen unter Druck setzen. Wir halten es für plausibel, dass eine andere Eigentümerstruktur in den Unternehmen zu steigenden und stärker kurzfristigen Profiterwartungen führt. Dieses wiederum könnte, vermittelt über Arbeitgeberverbände, zu Druck auf den Gesetzgeber führen, die Richtung eines „liberalen“ Regulierungssystems einzuschlagen. Solche Wirkungen der Mikroebene auf die institutionelle Ebene (Makroebene) geraten beim VoC-Ansatz leicht aus dem Blick.

Der zweite zutreffende Kritikpunkt lässt sich in der Formulierung von Heyes et al. (2012, S. 237) kondensieren: „VoC lacks a theory of capitalism“. Dieser Punkt ist insbesondere relevant, wenn wir wie hier nach den Auswirkungen der Krise auf die Eigentümerstrukturen und nach länderbzw. regulationssystemspezifisch unterschiedlichen Wirkungen fragen. Wir schließen daher Überlegungen der Marxistischen Krisentheorie an. Danach sind Krisen und zunehmender Wettbewerb Elemente eines fortschreitenden Akkumulationsprozesses, der „[...] stets mit Untergang vieler kleineren Kapitalisten, deren Kapitale teils in die Hand des Siegers übergehen, teils untergehn“ (Marx 1980, S. 655) verbunden ist. „Jede Akkumulation wird das Mittel neuer Akkumulation. Sie erweitert mit der vermehrten Masse des als Kapital funktionierenden Reichtums seine Konzentration in den Händen individueller Kapitalisten [...]“ (ebd. S. 653). Unter dieser Prämisse sollten wir nach der Finanzkrise aufgrund des verschärften Konkurrenzkampfes eine stärkere Eigentumskonzentration erwarten (Murray 2006; Murray/Scott 2012).

Neben Konzentrationsprozessen können sich Verschiebungen in der Zusammensetzung der Eigentümerschaft zeigen. Der 2008 manifest gewordenen Finanzkrise ging ein tief greifender, rund drei Jahrzehnte andauernder und nach wie vor anhaltender Prozess der Finanzialisierung voraus.
In diesem Prozess bekamen Finanzmärkte, Finanzinstitutionen und ihre Eliten zunehmend Einfluss auf die Wirtschaft (s. a. Harvey 2014). Diese Entwicklung führte zu einem Transfer von Einkommen von der Realwirtschaft hin zur Finanzwirtschaft. Während historisch gesehen die wesentliche Rolle von Finanzinstitutionen darin bestand, Geld gegen Zinsen zu verwalten und anderen Unternehmen Kredite zu gewähren, treten im Prozess der Finanzialisierung zunehmend Finanzinstitutionen als Anteilseigner auf. So wird sowohl in den USA als auch in Deutschland seit Langem diskutiert, ob sich der Einfluss der Banken im Laufe der Zeit verändert hat (vgl. für die USA: Herman 1981; Mizruchi/Domhoff 1982; für Deutschland: z. B. Beyer 2003; Streeck/Höpner 2003; Vitols 2004; Windolf 1994). Wir wollen daher den Einfluss der Banken vor und nach der Finanzkrise analysieren, indem wir die Veränderungen des Aktienbesitzes von Banken an den Top-200-Unternehmen betrachten. Wir vermuten insgesamt, dass die Wirkungen der Krise auf die Eigentümerstrukturen je nach Kapitalismustyp differieren. Zunächst werden wir deshalb darstellen, wie sich relevante Dimensionen je nach Typ des Kapitalismus unterscheiden. Wesentlich sind dabei vor allem die Art und Weise der Finanzierung der Unternehmen und die Rolle des Staates bei der Regulierung dieses Finanzierungssystems.

Die je nach System unterschiedliche Rolle des Staates ist von besonderer Bedeutung. Auch in liberalen Marktwirtschaften sind Unternehmen auf institutionelle Unterstützung angewiesen, etwa in Krisenzeiten auf staatlich finanzierte Konjunkturprogramme. Wir sollten solche Programme eher in koordinierten Marktwirtschaften mit ihrer stärkeren Bedeutung des Staates erwarten. Daraus folgt für unsere Frage nach den landesspezifischen Wirkungen der Krise die Vermutung, dass Krisen in koordinierten Marktwirtschaften, so auch in Deutschland, schwächer ausfallen und die Konzentrationseffekte und Finanzialisierungstendenzen geringer

TABELLE 1

\section{Finanzialisierung in Deutschland und den USA im Vergleich}

\begin{tabular}{|c|c|c|}
\hline \multirow[b]{2}{*}{ Indikatoren zur Unterscheidung von Finanzsystemen } & \multicolumn{2}{|c|}{ Deutschland } \\
\hline & 2003 & $2010 / 11$ \\
\hline Marktkapitalisierung börsennotierter Unternehmen (in \% des BIP) ${ }^{1}$ & 44,5 & 43,5 \\
\hline $\begin{array}{l}\text { Finanzstruktur (Verhältnis der Größe des Aktienmarktes relativ zum } \\
\text { Kreditvolumen der Banken) }{ }^{1}\end{array}$ & 0,31 & 0,33 \\
\hline Anteil des Vermögens in Pensionsfonds (in \% des BIP) ${ }^{2}$ & 9,2 & 13,3 \\
\hline $\begin{array}{l}\text { Bankaktiva in Staatsbesitz: Bankeinlagen in Banken, die mindestens zu } \\
50 \% \text { in staatlichem Besitz sind (in \% an allen Bankeinlagen) }(2011)^{3}\end{array}$ & 42 & 32 \\
\hline Aktienumschlag (Index; Wert aller gehandelten Aktien/Marktkapitalisierung) ${ }^{1}$ & 129,6 & 103,0 \\
\hline Veränderung des Aktienumschlags-Indizes in der Krise (2008 auf 2009) ${ }^{1}$ & \multicolumn{2}{|c|}{$193,3-107,2$} \\
\hline
\end{tabular}

\begin{tabular}{c|c}
\hline \multicolumn{2}{c}{ USA } \\
\hline $\mathbf{2 0 0 3}$ & $\mathbf{2 0 1 0 / 1 1}$ \\
\hline 128,6 & 118,9 \\
\hline 0,60 & 0,51 \\
\hline 70,3 & 69,2 \\
\hline 0 & 0 \\
\hline 122,6 & 189,1 \\
\hline \multicolumn{2}{|c|}{$404,1-348,6$} \\
\hline
\end{tabular}

Datenquellen: 1, 2 Weltbank (Global Financial Development Database GFDD; http://data.worldbank.org/data-catalog/global-financial-development); s .a. Čihák et al. (2012); zur Berechnung siehe Levine (2002); Hein (2005). 3 Barth et al. (2013).

Quelle: Zusammenstellung der Autoren. 
sind als in den USA. Dass der Finanzialisierungsgrad in liberalen Ökonomien höher ist als in koordinierten Marktwirtschaften, heißt konkret, dass in den USA ein größerer Anteil des Geldstroms über die Börse läuft, Aktien schneller umgeschlagen werden und wir häufiger reine Finanzanleger auf den Märkten finden. Auch dürfte der Staat eine geringere Rolle auf dem Finanzmarkt spielen. In Tabelle 1 haben wir einige Indikatoren zusammengestellt, die diese Unterschiede, die nach dem VoC-Ansatz zu erwarten sind, belegen.

Wir sehen, dass in den USA die Marktkapitalisierung börsennotierter Unternehmen höher ist (relativ zum Bruttoinlandsprodukt (BIP)). Im Jahr 2010 betrug dieser Wert $119 \%$ für die USA und $44 \%$ für Deutschland. Auch die Finanzstruktur unterscheidet sich: In den USA ist das Verhältnis des Aktienmarktes zum Kreditvolumen der Banken mit 0,51 deutlich höher als in Deutschland (0,33, jeweils für das Jahr 2010). Die Finanzierung von Unternehmen läuft also in den USA stärker über den Finanzmarkt im engeren Sinne und weniger über Bankkredite (van Treeck et al. 2007, S. 638, 640ff.). Der Vermögensanteil, den Pensionsfonds auf dem Finanzmarkt (relativ zum BIP) halten, ist in den USA höher (rund 69\% im Vergleich zu Deutschland mit 13\%). Der Anteil von Bankeinlagen in Banken, die sich mindestens zur Hälfte in Staatsbesitz befinden, beträgt in Deutschland (die Sparkassen und ähnliche Institute eingeschlossen) $32 \%$. In den USA ist dieser Anteil schlicht $0 \%$. Insgesamt zeigen die Indikatoren, dass sich die USA und Deutschland in ihrem Finanzsystem bzw. in ihrem Finanzialisierungsgrad deutlich unterscheiden.

Aufgrund dieser Systemunterschiede sollten wir ein an diese Systeme entsprechend angepasstes (und ein das System reproduzierendes) Verhalten der Unternehmen und eine andere Unternehmens,,landschaft“ beobachten können: Die Eigentümerstruktur in Deutschland dürfte weniger durch Finanzunternehmen geprägt und die Bedeutung der Banken als Aktienbesitzer sollte relativ zu anderen Formen des Finanzkapitals größer sein. Und wenn die Annahme zutrifft, dass Krisen die Konzentration erhöhen und Deutschland weniger von der Krise betroffen war, dann sollten wir hier eine geringere Zunahme der Konzentration vermuten als in den USA.

\subsection{Datenbasis}

Der von uns verwendete Datensatz basiert auf Informationen, die wir aus der OSIRIS-Datenbank (erstellt von dem Unternehmen Bureau van Dijk (BvD)) extrahiert und für unsere Fragen aufbereitet haben. Die kostenpflichtige Datenbank enthält Informationen über 65 Mio. Unternehmen weltweit mit Angaben über Aktiva, Marktkapitalisierung, Gewinn, Struktur der Anteilseigner und andere Kennzahlen.

In einem ersten Schritt haben wir für die USA und Deutschland jeweils die 200 umsatzstärksten börsennotierten Nicht-Finanzunternehmen (im folgenden Top-200 genannt) identifiziert und für die Zeiträume Juli 2006 bis Juni 2007 sowie für denselben Zeitraum 2009/2010 (also vor und nach der Krise) Informationen extrahiert. Wir betrachten hier das Eigentum an Nicht-Finanzunternehmen (Produktions- und Dienstleistungsunternehmen). Wir wollen die Eigentümer des produktiven Kerns der Ökonomien identifizieren und deren Zusammensetzung analysieren. Finanzunternehmen beziehen wir selbstverständlich als Eigentümer mit ein.

Das Geschäftsjahr (von Juli bis zum Juni des Folgejahres) haben wir gewählt, weil diese Festlegung bei einem Vergleich mehrerer Ländern mit unterschiedlichen Abgrenzungen eines Geschäftsjahres die größte zeitliche Überlappung liefert.

Wir verwenden Informationen über 221 Unternehmen in den USA und über 246 in Deutschland. Dass die Zahl mehr als 200 beträgt, liegt daran, dass wir die in dem jeweiligen Jahr umsatzstärksten Unternehmen zusammen einbezogen haben. Da wir die Strukturveränderungen der Unternehmen in ihrer Gesamtheit analysieren wollen, beziehen wir bewusst die Veränderungen der Zusammensetzung mit ein, die durch nach der Krise ausgeschiedene und neu hinzugekommene Unternehmen bewirkt werden. Dies wäre nicht möglich, wenn wir nur diejenigen Unternehmen erfassen würden, die zu beiden Zeitpunkten zu den Top-200 zählen. Wir nennen diese der Einfachheit weiterhin als „Top-200“ bezeichneten Unternehmen auch "Fokalunternehmen" - diese stehen im Fokus der Investoren, an ihnen halten sie Aktien.

In einem zweiten Schritt haben wir für jedes Fokalunternehmen jeden Anteilseigner identifiziert. Die Summe aller Aktien, die von einem Anteilseigner in einem Fokalunternehmen gehalten wird, bezeichnen wir als „Anteilseinheit“. Auf dieser Grundlage haben wir einen Datensatz gebildet, dessen Fälle die Anteilseinheiten sind. Unserer Datensatz enthält insgesamt 35.835 Beobachtungen über die Eigentümer der Top-200; dabei entfallen 22.749 Anteilseinheiten auf die US-Fokalunternehmen und 13.086 auf die deutschen. Dabei betrachten wir nur Anteilseinheiten, die mindestens ein Prozent des Börsenwertes eines Unternehmens ausmachen und die wir deswegen als „signifikant” bezeichnen. Anteilseigner, die weniger als ein Prozent an den Anteilen halten, haben kaum Möglichkeiten, auf die Geschäfte des fokalen Unternehmens Einfluss zu nehmen. Wenn wir den Schwellenwert von einem Prozent zugrunde legen, erfassen wir in jedem der beiden Länder etwa zwei Drittel der Anteile an allen Nicht-Finanzunternehmen.

\section{Wer sind die Eigentümer?}

In Tabelle 2 sind die zehn größten Eigentümer der fokalen Unternehmen für die beiden Länder und die beiden Jahre dargestellt. Wir haben für beide Länder für jedes Unternehmen berechnet, welchen Anteil es an den Top-200 insgesamt hält und eine entsprechende Rangfolge gebildet. Die zehn Unternehmen mit den höchsten Anteilen bezeichnen wir 
TABELLE 2

Top-10-Anteilseigner in den USA und Deutschland, 2006/07 und 2009/10 Angaben in Prozent

\begin{tabular}{lc|c|c}
\hline & \multicolumn{3}{c}{ USA } \\
\cline { 2 - 4 } & $\mathbf{2 0 0 6 / 2 0 0 7}$ & $\mathbf{2 0 0 9 / 2 0 1 0}$ & Veränderung gesamt \\
\hline BlackRock & 9,4 & 13,1 & 3,7 \\
\hline Capital Group & 9,6 & 9,9 & 0,3 \\
\hline State Street Corporation & 9,4 & 9,4 & 0,0 \\
\hline Vanguard Group & 6,1 & 8,0 & 1,9 \\
\hline Fidelity Group & 6,6 & 5,3 & $-1,3$ \\
\hline Wellington Management & 3,5 & 3,1 & $-0,4$ \\
\hline Bank of New York & 2,8 & 3,1 & 0,3 \\
\hline Mellon Corporation & 2,9 & 3,2 & 0,3 \\
\hline Northern Trust Corporation & 2,7 & 3,2 & 0,5 \\
\hline Walton Enterprises & 1,3 & 2,4 & 1,1 \\
\hline T. Rowe Price Group & 54,1 & 60,7 & 6,6 \\
\hline Summe der von den Top-10 & & & \\
\hline gehaltenen Aktienanteile & & & \\
\hline
\end{tabular}

\begin{tabular}{lc|c|c}
\hline & \multicolumn{3}{c}{ Deutschland } \\
\cline { 2 - 4 } & $\mathbf{2 0 0 6 / 2 0 0 7}$ & $\mathbf{2 0 0 9 / 2 0 1 0}$ & Veränderung gesamt \\
\hline Volkswagen AG & 6,9 & 6,8 & $-0,1$ \\
\hline Capital Group & 5,5 & 6,4 & 0,9 \\
\hline BlackRock & - & 5,0 & 5,0 \\
\hline Porsche Automobil Holding SE & 2,0 & 4,6 & 2,6 \\
\hline Deutsche Bank AG & 2,5 & 3,4 & 0,9 \\
\hline Bundesanstalt für & 2,4 & 2,8 & 0,4 \\
\hline Post u. Telekommunikation & 2,1 & 2,8 & 0,7 \\
\hline Familien: Beisheim; Haniel; & & 2,7 & 2,7 \\
\hline Schmidt-Ruthenbeck & 0,0 & 2,6 & 0,4 \\
\hline Regierung von Norwegen & 2,2 & 2,1 & 1,6 \\
\hline Allianz SE & 0,5 & 39,2 & 15,1 \\
\hline Barclays & 24,1 & & \\
\hline Summe der von den Top-10 & & & \\
\hline gehaltenen Aktienanteile & & & \\
\hline
\end{tabular}

Anmerkung: kursiv hervorgehoben sind diejenigen Unternehmen, die in beiden Ländern als Anteilseigner vertreten sind.

als Top-10. So hält z. B. BlackRock 2009/2010 in den USA $13 \%$ der Anteilseinheiten an allen Top-200-Unternehmen. BlackRock Inc., ein Finanz- und Fondsmanagement-Unternehmen mit Sitz in New York, ist damit der wichtigste Aktienkontrolleur in den USA. Die Medien bezeichnen das Unternehmen als „Besitzer der Welt“ (Unterreiner 2011) oder als „heimliche Weltmacht“ (Buchter 2015). Immerhin verwaltet BlackRock für seine Kunden nach eigenen Angaben US\$ 4,6 Bill. (http://www.blackrock.com/corporate/en-de/ about-us für 2014), ein Volumen, das um einiges größer ist als das Bruttoinlandsprodukt von Deutschland für das Jahr 2015. Das Wachstum von BlackRock ist rasant: Vor der Krise (20072008) betrug die verwaltete Summe rund 1,3 Bill.; 2009 waren es bereits US\$ 3,3 Bill. (BlackRock 2009).
Neben BlackRock findet sich unter den Eigentümern in den USA eine Mischung aus Banken, Finanzunternehmen und Investmentfonds. Zudem enthält unsere Liste der Top10 die Walton-Familie, eine der reichsten Familien der Welt, deren Eigentum an den Wal-Mart-Märkten von Forbes (Worstall 2011) auf rund US\$ 93 Mrd. geschätzt wird.

In Deutschland ist die Eigentümerstruktur erstens anders als in den USA - durch eine recht große Anzahl von Individuen und Familien charakterisiert. Die Volkswagen AG ist eine der zentralen Eigentümerinnen und das größte fokale Unternehmen. Diese Positionierung beruht wesentlich auf dem $99 \%$ igen Anteil an Audi und dem $30 \%$-Anteil an MAN.

Auffällig ist zweitens, dass BlackRock und Capital Group nicht nur in ihrem Stammland, den USA, sondern auch in Deutschland eine große Rolle spielen. Nur diese beiden Unternehmen sind als Eigentümer in beiden Ländern unter den ersten Zehn vertreten. Zwar ist BlackRocks Präsenz in seinem Ursprungsland, den USA, am stärksten. Allerdings ist BlackRock in Deutschland an allen DAX-Konzernen beteiligt (Kremer 2013). Sowohl BlackRock als auch die Capital Group haben ihren Anteil in beiden Ländern deutlich erhöht. Beide zusammen halten in Deutschland $11 \%$ und in den USA sogar $23 \%$ der Aktienanteile der Top-200.

Wir finden in Tabelle 2 auch Hinweise auf das Ausmaß und auf Veränderungen in den Konzentrationsgraden des Eigentums. In den USA liegt der Anteil, den die Top-10 an den fokalen Unternehmen halten, gerundet bei 54 bzw. $61 \%$. Dieser hat während der Krise leicht zugenommen. In Deutschland ist der Anteil der Top-10 mit 24 bzw. 39\% geringer, die Zunahme der Konzentration ist aber stärker ausgefallen.

\section{Anlagestrategien}

Die unterschiedlichen Gruppen von Kapitaleignern differieren in ihren Investitions- und Kontrollstrategien. Der Aktienbesitz von Individuen, Familien oder Industrieund Dienstleistungsunternehmen ist oftmals groß, aber nur auf ein oder wenige (börsennotierte) Unternehmen beschränkt. Dagegen kontrolliert das Finanzkapital häufiger eher kleine Anteile, dafür aber in sehr vielen Unternehmen (Peetz/Murray 2012). Im Folgenden gehen wir der Frage nach, in welchem Ausmaß Anteilskapitaleigner eine dominante Position einnehmen, das heißt, der größte Anteilseigner in einer großen Anzahl von fokalen Unternehmen zu sein. Eine andere Strategie könnte darin bestehen, eine dominante Position entweder nicht anzustreben oder diese nur auf wenige fokale Unternehmen zu konzentrieren (wir sprechen dann von intensiver oder fokussierter Dominanz; siehe zu den operationalen Definitionen Tabelle 3). 
Interessant ist eine Antwort auf die Frage nach den Strategieunterschieden innerhalb der und zwischen den beiden Ländern. Ausgehend vom VoC-Ansatz sollten wir für die USA eine stärker marktorientierte Streuung und damit weniger eine Strategie der Fokussierung vorfinden.

In den Tabellen 3 und 4 konzentrieren wir uns auf die jeweiligen TOP-30-Anteilseigner in den beiden Ländern. Wir berechnen für jedes dieser Unternehmen, in wie vielen Fokalunternehmen es Aktienanteile hält und inwieweit es zu den größten bzw. großen Anlegern des jeweiligen Landes gehört. Damit wollen wir erfassen, welches Gewicht die Anteilseigner auf Dominanz legen, also darauf, der größte oder zweitgrößte Anleger in dem fokalen Unternehmen zu sein. Die Grenzen, die wir für die Operationalisierung zwischen sehr starker, starker usw. Dominanz ziehen, sind pragmatisch gewählt. Ein Beispiel zur Erläuterung: Ein sehr stark dominanter Anteilseigner ist nach unserer Definition ein Unternehmen, das entweder

- bezogen auf seine Anlagen insgesamt mindestens $10 \%$ Anteilseinheiten hält, die in den fokalen Unternehmen

TABELLE 3

\section{Anlagestrategien in den USA, 2009/2010}

Angaben in absoluten Zahlen und in Prozent

\begin{tabular}{|c|c|c|c|c|c|c|}
\hline \multirow[b]{2}{*}{ Dominanz der Anteilseigner* } & \multirow[b]{2}{*}{$\begin{array}{l}\text { Anzahl von fokalen } \\
\text { Unternehmen, in } \\
\text { denen die Anteils- } \\
\text { eigner Aktien } \\
\text { halten }\end{array}$} & \multicolumn{5}{|c|}{$\begin{array}{l}\text { Prozent des Anteils an Unternehmen, in denen der } \\
\text { Anteilseigner der größte, zweitgrößte, dritt- bis } \\
\text { fünftgrößte usw. Anleger ist }\end{array}$} \\
\hline & & $\begin{array}{c}1 \\
\text { (größter } \\
\text { Anleger) }\end{array}$ & $\begin{array}{c}\quad 2 \\
\text { (zweit- } \\
\text { größter } \\
\text { Anleger) }\end{array}$ & 3 bis 5 & 6 bis 10 & $11+$ \\
\hline \multicolumn{2}{|l|}{ sehr starke Dominanz } & \multicolumn{5}{|c|}{ in $\%$} \\
\hline BlackRock, Inc. & 208 & 29,3 & 31,7 & 35,1 & 1,9 & 1,9 \\
\hline Capital Group Companies & 150 & 24,0 & 20,0 & 18,0 & 16,7 & 21,3 \\
\hline Fidelity Group & 195 & 10,3 & 9,2 & 20,5 & 22,6 & 37,4 \\
\hline \multicolumn{7}{|l|}{ eher starke Dominanz } \\
\hline State Street Corporation & 203 & 6,4 & 7,4 & 47,8 & 30,0 & 8,4 \\
\hline \multicolumn{7}{|l|}{ eher geringe Dominanz } \\
\hline AXA & 201 & 3,0 & 2,0 & 8,5 & 15,9 & 70,6 \\
\hline Franklin Resources, Inc. & 128 & 2,3 & 2,3 & 7,8 & 15,6 & 71,9 \\
\hline Wellington Management Co L.L.P. & 143 & 2,1 & 5,6 & 13,3 & 36,4 & 42,7 \\
\hline Vanguard Group, Inc. & 188 &, 5 & 5,3 & 64,4 & 28,7 & 1,1 \\
\hline T. Rowe Price Group, Inc. & 184 &, 5 & 2,7 & 10,9 & 21,7 & 64,1 \\
\hline \multicolumn{7}{|l|}{ sehr geringe Dominanz } \\
\hline Bank of New York Mellon Corporation & 202 & 0,5 & 0,0 & 3,0 & 34,2 & 62,4 \\
\hline Janus Capital Group, Inc. & 160 & 0,0 & 1,9 & 3,1 & 10,0 & 85,0 \\
\hline JP Morgen Chase \& Co. & 204 & 0,0 & 0,0 & 6,4 & 18,1 & 75,5 \\
\hline Invesco Ltd. & 193 & 0,0 & 0,0 & 2,6 & 16,1 & 81,3 \\
\hline Ameriprise Financial, Inc. & 197 & 0,0 & 0,0 & 2,5 & 12,2 & 85,3 \\
\hline Northern Trust Corporation & 202 & 0,0 & 0,0 & 1,5 & 29,2 & 68,8 \\
\hline Wells Fargo \& Company & 200 & 0,0 & 0,0 & 1,0 & 8,0 & 91,0 \\
\hline Bank of America Corporation & 198 & 0,0 & 0,0 & 1,0 & 5,6 & 93,4 \\
\hline $\begin{array}{l}\text { Teachers Insurance \& Annuity Association } \\
\text { of America }\end{array}$ & 202 & 0,0 & 0,0 & 0,5 & 4,5 & 95,0 \\
\hline \multicolumn{7}{|l|}{ fokussierte Dominanz } \\
\hline Walton Enterprises LLC & 1 & 100,0 & 0,0 & 0,0 & 0,0 & 0,0 \\
\hline Page Larry & 1 & 100,0 & 0,0 & 0,0 & 0,0 & 0,0 \\
\hline
\end{tabular}

*Definitionen: (a) Sehr stark dominante Anteilseigner halten entweder mindestens $10 \%$ Anteile, die in den fokalen Unternehmen den größten Anteil (den ersten Platz im Ranking der Anteile) darstellen, oder mindestens $20 \%$ an Anteilen auf dem ersten oder zweiten Platz erfassen; (b) eher starke Dominanz: entweder mindestens $4 \%$ der Anteile auf Platz 1 oder $8 \%$ auf Platz 1 oder 2, aber nicht in Gruppe a; (c) eher geringe Dominanz: das Unternehmen ist nicht in Gruppe a, b oder d; (d) sehr geringe Dominanz: $1 \%$ oder weniger Anteile, die auf Platz 1 in den fokalen Unternehmen stehen, und $2 \%$ oder weniger auf Platz 1 oder 2; (e) oder d; (d) sehr geringe Dominanz: $1 \%$ oder weniger Anteile, die auf Platz 1 in den fokalen Unternehme 
den größten Anteil haben und damit den ersten Platz im Ranking der Anteile einnehmen, oder

- mindestens $20 \%$ an Anteilen hält, die auf dem ersten oder zweiten Platz des Fokalunternehmens einzugruppieren sind.

Die Befunde für die USA (Tabelle 3) zeigen: BlackRock hält Anteile in 208 (von insgesamt 221) fokalen US-Unternehmen. In $29 \%$ dieser Unternehmen ist BlackRock jeweils der Anleger mit dem größten Anteil. In weiteren $32 \%$ ist BlackRock der zweitgrößte Anleger (in 35\% ist BlackRock der dritt- bis fünftgrößte). Anders gesagt: in $95 \%$ von 208 Unternehmen ist BlackRock unter den fünf großen Anlegern. BlackRock repräsentiert damit eine Anlagestrategie, die wir hier als ,sehr starke extensive Dominanz" bezeichnen.
Auch die Capital Group verfolgt diese Strategie: Die Unternehmensgruppe hält in 150 (von 221) Fokalunternehmen Anlagen, in $44 \%$ dieser Unternehmen ist Capital Group der größte oder zweitgrößte Aktionär (in $62 \%$ mindestens der fünftgrößte). In abgeschwächtem Maße gilt diese Strategie auch für die Fidelity Group: Diese Unternehmensgruppe hält Anlagen in sehr vielen Fokalunternehmen, in $40 \%$ ist sie mindestens unter den fünftgrößten Anlegern. Eine Strategie der starken Dominanz gibt diesen Anlegern die Möglichkeit, das Verhalten sehr vieler Fokalunternehmen zu beeinflussen.

Am anderen Ende unserer Strategieskala (Tabelle 3) finden wir Unternehmen, die nicht dominant sind und keine Führungsrolle übernehmen, genauer: die ihre Anlagen breiter streuen und Anteile in sehr vielen Unternehmen halten,

TABELLE 4

Anlagestrategien in Deutschland, 2009/2010

Angaben in absoluten Zahlen und in Prozent

\begin{tabular}{|c|c|c|c|c|c|c|}
\hline \multirow[b]{2}{*}{ Dominanz der Anteilseigner } & \multirow[b]{2}{*}{$\begin{array}{l}\text { Anzahl von fokalen } \\
\text { Unternehmen, } \\
\text { in denen die } \\
\text { Anteilseigner } \\
\text { Aktien halten }\end{array}$} & \multicolumn{5}{|c|}{$\begin{array}{c}\text { Prozent des Anteils an Unternehmen, in denen } \\
\text { der Anteilseigner der größte, zweitgrößte, dritt } \\
\text { bis fünftgrößte usw. Anleger ist }\end{array}$} \\
\hline & & $\begin{array}{l}1 \\
\text { (größter } \\
\text { Anleger) }\end{array}$ & $\begin{array}{c}2 \\
\text { (zweit- } \\
\text { größter } \\
\text { Anleger) }\end{array}$ & 3 bis 5 & 6 bis 10 & $11+$ \\
\hline \multicolumn{2}{|l|}{ eher starke Dominanz } & \multicolumn{5}{|c|}{ in $\%$} \\
\hline Capital Group & 56 & 5,4 & 8,9 & 26,8 & 25,0 & 33,9 \\
\hline BlackRock & 94 & 5,3 & 5,3 & 19,1 & 29,8 & 40,4 \\
\hline \multicolumn{7}{|l|}{ eher geringe Dominanz } \\
\hline Deutsche Bank AG & 115 & 1,7 & 7,0 & 27,0 & 24,3 & 40,0 \\
\hline Franklin Resources, Inc. & 29 & 0,0 & 10,3 & 24,1 & 20,7 & 44,8 \\
\hline Allianz SE & 113 & 0,0 & 5,3 & 13,3 & 39,8 & 41,6 \\
\hline DekaBank & 80 & 0,0 & 2,5 & 7,5 & 18,8 & 71,3 \\
\hline \multicolumn{7}{|l|}{ sehr geringe Dominanz } \\
\hline Government of Norway & 99 & 1,0 & 1,0 & 15,2 & 45,5 & 37,4 \\
\hline Barclays PLC & 85 & 0,0 & 1,2 & 4,7 & 29,4 & 64,7 \\
\hline Government of Qatar & 1 & 0,0 & 0,0 & 100,0 & 0,0 & 0,0 \\
\hline \multicolumn{7}{|l|}{ fokussierte Dominanz } \\
\hline RWE AG & 3 & 100,0 & 0,0 & 0,0 & 0,0 & 0,0 \\
\hline Volkswagen AG & 2 & 100,0 & 0,0 & 0,0 & 0,0 & 0,0 \\
\hline Porsche Automobil Holding SE & 2 & 100,0 & 0,0 & 0,0 & 0,0 & 0,0 \\
\hline KfW Bankengruppe & 1 & 100,0 & 0,0 & 0,0 & 0,0 & 0,0 \\
\hline Siemens-Belegschaftsaktionaere & 1 & 100,0 & 0,0 & 0,0 & 0,0 & 0,0 \\
\hline Maxingvest AG & 1 & 100,0 & 0,0 & 0,0 & 0,0 & 0,0 \\
\hline Familien Haniel & 1 & 100,0 & 0,0 & 0,0 & 0,0 & 0,0 \\
\hline Aabar Investments PJSC & 1 & 100,0 & 0,0 & 0,0 & 0,0 & 0,0 \\
\hline Plattner, Hasso & 1 & 100,0 & 0,0 & 0,0 & 0,0 & 0,0 \\
\hline Henkel Family & 1 & 100,0 & 0,0 & 0,0 & 0,0 & 0,0 \\
\hline Land Niedersachsen & 2 & 50,00 & 50,00 & 0,0 & 0,0 & 0,0 \\
\hline
\end{tabular}

Anmerkung: Definitionen siehe Tabelle 3.

Quelle: Berechnungen der Autoren auf Basis der BvD-OSIRIS-Datenbank. 
aber in fast allen dieser Fokalunternehmen nicht zu den beiden großen Anteilseignern gehören. Ein Beispiel ist JPMorgan Chase, die in mehr als 200 US-Unternehmen Anteile besitzen, aber in keinem einzigen der Fokalunternehmen zu den beiden größten Anlegern gehören. Auch die Bank of New York Mellon verfolgt diese nicht-dominante Strategie, obwohl diese Bank zu den zehn großen Anlegern in den USA zählt.

Zwischen der Strategie einer sehr starken und sehr geringen Dominanz sind diejenigen Anleger einzuordnen, die wir hier als „mittel“ (als eher stark oder eher gering) eingruppiert haben.

Schließlich gibt es unter den 30 großen Anlegern eine kleine Gruppe, die wir als „, fokussiert dominante“ Anleger bezeichnen; oftmals Individuen oder Familien, die nur in einem oder zwei Unternehmen Anteile halten, in diesen aber der dominierende Anteilseigner sind. In den USA sind dies Walton Enterprises und Larry Page (Google).

Betrachten wir nun Deutschland (Tabelle 4) und vergleichen die Anlegerstrategien mit denen, die wir in den USA vorfinden. Der Vergleich zeigt erstens, dass es in Deutschland keinen Anleger mit einer stark extensiven Dominanz gibt. Zweitens fällt auf, dass die beiden größten Anleger - BlackRock mit Sitz in New York und Capital Group mit Sitz in Los Angeles -, die in den USA eine Strategie der extensiven Dominanz verfolgen, auch in Deutschland die beiden stärksten sind. Ihre Strategie ist für diese beiden Länder konsistent bzw. global. Drittens: Die fokussierten, intensiv dominanten Anleger bilden in Deutschland die Mehrheit innerhalb der Top-30, während sie in den USA die Minderheit darstellen. Die Anteilseigner-Familien und -Individuen sind in Deutschland stärker und Finanzunternehmen schwächer vertreten als in den USA.

\section{Konzentration}

Bereits die namentliche Analyse der Eigentümer deutete auf eine erhebliche Konzentration hin. Ein weiterer Indikator zur Messung von Konzentration ist der Anteil des Aktienwertes, der von den Top-N (den größten zwei, vier etc.) Unternehmen kontrolliert wird (Tabelle 5). 2009/2010 hielten die beiden größten Aktionäre (Top-2) in den USA $23 \%$ der signifikanten Anteile (19\% in 2006/2007). Die Top-7 besaßen 2009/10 52\% der signifikanten Aktien, während es in 2006/2007 noch $48 \%$ waren. Bezogen auf die Top-20 zeigten sich mit rund $71 \%$ kaum Unterschiede. Die Zunahme der Konzentration geht in den USA also auf die großen Anteilseigner zurück.

In Deutschland hat die Konzentration dagegen nicht nur bei den großen Anteilseignern zugenommen, sondern über alle Gruppen hinweg. Gleichwohl ist das Gesamtausmaß der Konzentration um einiges geringer als in den USA. Während in den USA die Top-20 71\% der signifikanten Anteile halten, sind es in Deutschland „nur“ $56 \%$.

\section{Verschiebungen hin zum Finanzkapital?}

Zunächst vergleichen wir die beiden Länder hinsichtlich der Bedeutung von Anteilseignern, die wir als Finanzkapital bezeichnen, im Verhältnis zu anderen Anlegern. Wir verstehen unter Finanzkapital Unternehmen, die von BvD als Finanzunternehmen, Pensionsfonds o. ä., Banken, Versicherungen oder Private Equity Firms kategorisiert werden (Tabelle 6).

Der auffälligste Unterschied zwischen den beiden Ländern liegt im Anteil des Aktienswertes, den das Finanz-

TABELLE 5

\section{Signifikante Anteile der Top-N Anteilseigner an allen signifikanten Anteilseinheiten in den USA und Deutschland, 2006/2007 und 2009/2010}

Angaben in Prozent

\begin{tabular}{l|c|c|c|}
\hline Top-N & \multicolumn{3}{|c}{ USA } \\
\hline & $\mathbf{2 0 0 6 / 0 7}$ & $\mathbf{2 0 0 9 / 1 0}$ & Veränderung gesamt \\
\hline Top-2 & 18,7 & 23,0 & 4,3 \\
\hline Top-4 & 34,5 & 40,4 & 5,9 \\
\hline Top-7 & 47,7 & 51,9 & 4,2 \\
\hline Top-10 & 55,9 & 60,7 & 4,8 \\
\hline Top-15 & 65,7 & 67,7 & 2,0 \\
\hline Top-20 & 72,4 & 71,1 & $-1,3$ \\
\hline
\end{tabular}

\begin{tabular}{c|c|c|}
\hline \multicolumn{3}{|c}{ Deutschland } \\
\hline $\mathbf{2 0 0 6 / 0 7}$ & $\mathbf{2 0 0 9 / 1 0}$ & Veränderung gesamt \\
\hline 12,4 & 13,2 & 0,8 \\
\hline 18,8 & 22,7 & 3,9 \\
\hline 26,5 & 32,2 & 5,7 \\
\hline 33,4 & 40,3 & 6,9 \\
\hline 43,4 & 49,4 & 6,0 \\
\hline 51,3 & 56,3 & 5,0 \\
\hline
\end{tabular}


TABELLE 6

Eigentum signifikanter Aktienanteile in den Top-200 in den USA und in Deutschland nach Art der Aktieneigentümer, 2006/2007 und 2009/2010

Angaben in Prozent

\begin{tabular}{|c|c|c|c|c|c|c|}
\hline & & & & Det & and & \\
\hline & 2006/07 & $2009 / 10$ & $\begin{array}{c}\text { Veränderung } \\
\text { gesamt }\end{array}$ & 2006/07 & $2009 / 10$ & $\begin{array}{c}\text { Veränderung } \\
\text { gesamt }\end{array}$ \\
\hline Banken & 27,5 & 22,7 & $-4,8$ & 16,3 & 16,3 & 0,0 \\
\hline andere Finanzunternehmen & 57,0 & 60,1 & 3,1 & 23,4 & 25,3 & 1,9 \\
\hline Finanzkapital insgesamt & 84,5 & 82,8 & $-1,7$ & 39,7 & 41,6 & 1,9 \\
\hline Individuen oder Familien (namentlich genannt) & 5,0 & 8,9 & 3,9 & 13,1 & 10,2 & $-2,9$ \\
\hline Industrie- und Dienstleistungsunternehmen & 9,5 & 6,9 & $-2,6$ & 34,1 & 33,3 & $-0,8$ \\
\hline öffentliche Institutionen (Staaten, Regierungen etc.) & * & 0,2 & 0,2 & 7,6 & 8,1 & 0,5 \\
\hline andere & 1,0 & 1,1 & 0,1 & 5,3 & 6,3 & $-1,0$ \\
\hline signifikanter Aktienbesitz insgesamt & 100 & 100 & & 100 & 100 & \\
\hline Anzahl von Top-Fokalunternehmen It. BvD-Datenbank & & & 221 & & & 246 \\
\hline Anzahl von Aktienanteilseinheiten It. BvD-Datenbank & & & 22.749 & & & 13.086 \\
\hline
\end{tabular}

* Weniger als $0,1 \%$.

Quelle: Berechnungen der Autoren auf Basis der BvD-OSIRIS-Datenbank.

kapital an den Top-200-Unternehmen hält. In den USA beträgt dieser Anteil $85 \%$, während er in Deutschland mit rund $42 \%$ deutlich niedriger liegt. Nach wie vor spielen Industrie- und Dienstleistungsunternehmen, aber auch Individuen und Familien als Anleger in Deutschland eine wichtigere Rolle als in den USA, allerdings nimmt deren Bedeutung, zumindest im Untersuchungszeitraum, leicht ab. Zudem besitzen staatliche Anleger in Deutschland einen größeren Anteil der Aktien als in den USA.

Für die USA zeigt sich in den Daten eine leichte Verschiebung von den Banken hin zu anderen Finanzkapital-Gruppen. In den Jahren 2009/2010 wurde der größte Anteil der signifikanten US-Aktien (60,1\%) von Finanzunternehmen (Nicht-Banken) kontrolliert, während Banken $22,7 \%$ hielten. Andere Typen von Eigentümern - andere Industrie- und Dienstleistungsunternehmen, Individuen oder Familien - besitzen nur einen geringen Anteil der Aktien. Der Anteil, den namentlich genannte Eigentümer halten, hat in den USA etwas zugenommen, dies ist u. a. auf die größere Bedeutung von Larry Page (Google) zurückzuführen. Der Anteil der Banken ist in den USA wenig zurückgegangen. Staatliche Eingriffe zur Bankenrettung durch Eigenkapitalzufuhr haben die Eigentümerstruktur kaum verändert. Zwar mussten in den USA während der Finanzkrise etliche Banken schließen. Allerdings betraf dies bis auf eine Ausnahme (Lehman Brothers) nur kleinere Banken. Alle anderen wurden (auch gegen den Willen des Steuerzahlers) gerettet. Dies dürfte der Grund dafür sein, dass die Banken ihre Anteile an den großen Unternehmen weiter halten konnten.
In Deutschland wurde nicht nur ein deutlich geringerer (etwa halb so großer) Anteil der Aktien vom Finanzkapital gehalten. Im Untersuchungszeitraum war etwa ein Drittel aller Anteile in den Fokalunternehmen im Besitz von Nicht-Finanzunternehmen. Allerdings sank dieser Anteil, und es zeigte sich eine Zunahme der auf das Finanzkapital insgesamt entfallenden Anteile um 1,9 Prozentpunkte. In Deutschland gab es anders als in den USA keine Veränderung des von Banken gehaltenen Aktienanteils. ${ }^{4}$

In der am stärksten von Finanzialisierung geprägten Volkswirtschaft, in den USA, hat die Finanzkrise nicht zu einer Verstärkung dieses Prozesses geführt. Möglicherweise ist bereits ein Sättigungspunkt der Finanzialisierung erreicht. Die Bedeutung des Finanzkapitals hat Deutschland - in einem von der Krise geringer betroffenen Land - vergleichsweise stärker zugenommen. Aber auch hier ist die Veränderung insgesamt schwach ausgeprägt. Allerdings können wir nur einen kurzen Zeitraum betrachten. Würden sich die Veränderungen über einen längeren (bspw. von zehn Jahren) aufsummieren, wären sie keineswegs mehr gering.

4 Die Deutsche Bundesbank (2014, S. 19) kommt zu dem Befund, dass die deutschen Banken während der Krise „ihr Engagement in deutschen Aktien reduziert" haben. Allerdings macht der Bericht nur Aussagen über die inländischen Anleger. Zudem ist die Unterscheidung zwischen den Investorengruppen anders als in der BVD-Datenbank und kaum vergleichbar. 


\section{Schlussfolgerungen}

Fassen wir zunächst die Ergebnisse knapp zusammen. Unsere erste Frage war, welche Unternehmen die größten Anteile an den Aktien anderer (Nicht-Finanz-)Unternehmen besitzen. Auffällig ist hier die Stellung des Vermögensverwalters BlackRock, aber auch der Capital Group. Beide kontrollieren sowohl in den USA als auch in Deutschland einen bedeutenden Anteil des Aktienkapitals; für beide Unternehmen können wir während der Krise eine Zunahme beobachten. Zweitens wollten wir wissen, welche Anlagestrategien die Anleger verfolgen. Wir können feststellen, dass Deutschlands Top-30-Anleger stärker als die in den USA eine fokussiert dominante Strategie verfolgen, sich also auf wenige Unternehmen konzentrieren und in diesen dann mindestens $50 \%$ der Anteile halten. Diese Beobachtung korrespondiert mit einem Muster, das wir vor dem Hintergrund des VoC-Ansatzes erwarten sollten. Unsere dritte Frage richtete sich auf die Konzentration des signifikanten Aktienbesitzes. Die Befunde sprechen für eine höhere Konzentration bezogen auf den Besitz an den Fokalunternehmen in den USA. Zum einen hat der Anteil, der auf die Top-2-bzw. Top-7-Unternehmen entfällt, zugenommen. Zum anderen ist bemerkenswert, dass in über $70 \%$ der 221 US-Unternehmen entweder BlackRock oder Capital Group (oder beide) den größten oder zweitgrößten Anteil halten. Weiterhin hat die Konzentration in den USA insbesondere bei den Top-10 stärker zugenommen als in Deutschland. Offenbar hat die Krise Raum geschaffen für Konzentrationsprozesse. Viertens hatten wir nach der Möglichkeit einer Verschiebung des Aktienbesitzes hin zum Finanzkapital gefragt. Eine solche Entwicklung würden wir als Hinweis auf eine stärkere Finanzialisierung interpretieren. Allerdings kann man jedenfalls bezogen auf den kurzen Analysezeitraum - in keinem der beiden Länder einen massiven Finanzialisierungsschub beobachten. Gleichwohl sehen wir zum einen eine Fortsetzung des starken Einflusses des Finanzkapitals, vor allem in den USA, dies trotz oder wegen der zentralen Rolle des Finanzkapitals als Verursacher der Krise. Zum anderen beobachten wir in den USA eine leichte Verschiebung weg von denjenigen Subgruppen des Finanzkapitals, die am engsten mit der Krise assoziiert sind, nämlich den Banken, wobei auffallend ist, wie gering der Rückgang des Anteils der Banken ist. Wir sehen eine mögliche Ursache für diese relative Stabilität darin, dass die Banken „too big to fail" sind, als systemrelevant eingeschätzt werden und möglicherweise auch politischer Einfluss durch die Banken ausgeübt wurde (dies ist selbstverständlich eine Deutung, die einen interpretativen Überschuss über unsere Empirie aufweist).

Die Befunde bestätigen, dass sich das Eigentum am Vermögen der realwirtschaftlich produktiven Unternehmen in „wenigen Händen“ und zudem beim Finanzkapital konzentriert. In den USA halten die zehn größten Anleger rund
$61 \%$ des realwirtschaftlichen Aktienvermögens, in Deutschland $39 \%$. Die Finanzinvestoren BlackRock und Capital Group besitzen in den USA $23 \%$ und in Deutschland $11 \%$ dieser Aktienanteile.

Die Befunde werfen die Frage auf, was die beschriebenen Veränderungen für das Verhalten von Unternehmen bedeuten und welche Folgen für die Beschäftigten zu erwarten sind.

Zugespitzt formuliert kann man sagen, dass die beiden Formen des Kapitalismus nach unterschiedlichen Logiken funktionieren. In Deutschland sehen wir unseren Daten zufolge eine Logik, die wir hier als Elitefamilien- und Industriekapitalismus bezeichnen. Diese Logik manifestiert sich in den Anlagestrategien der Nicht-Finanzunternehmen, insbesondere der Industrie- und Dienstleistungsunternehmen und reicher Eigentümerfamilien in Deutschland. $\mathrm{Zu}$ vermuten ist, dass nach dieser Logik auch Inhaber-Persönlichkeiten stärker als in anderen Unternehmen darüber entscheiden, ob eher eine Strategie der kurzfristigen oder längerfristigen Gewinnmaximierung verfolgt oder eher eine nachhaltige Wachstumsstrategie angestrebt wird. In den USA treibt dagegen die Finanzlogik - der Finanzkapitalismus - auch die Nicht-Finanzunternehmen. Dies würde dafür sprechen, dass in den USA das Ziel kurzfristiger Aktiengewinne eine größere Rolle spielt (Peetz/Murray 2012), während dieses Ziel in Deutschland eine relativ schwächere Bedeutung hat. Gleichwohl ist zu erwarten, dass auch in Deutschland die Veränderungen auf der Ebene der Eigentümerstrukturen in den Unternehmen mittelfristig nicht folgenlos bleiben werden. Eine stärkere Dominanz von Anlegern wie BlackRock und ähnlichen Unternehmen dürfte zu einer Veränderung in Richtung steigender Profiterwartungen führen (Windolf 2013). Dies kann Forderungen an den Gesetzgeber motivieren, nicht nur das Finanzsystem, sondern auch die anderen institutionellen Pfeiler, insbesondere in koordinierten Marktwirtschaften, weiter zu liberalisieren, etwa in Form von Druck auf die Deregulierung von Arbeitsmärkten oder in Richtung auf einen Rückbau des Wohlfahrtsstaates. Beides hat langfristig Konsequenzen für die Verhandlungsposition der Arbeitnehmerseite. Zweitens, und dies ist eine eher kurzfristige Folge, werden sich insbesondere in solchen Unternehmen, in denen institutionelle Anleger wie BlackRock aktiv sind, die Unternehmensleitungen stärker an kurzfristigen, finanziellen Steuerungsgrößen ausrichten (Kennzahlenorientierung). Dies wird sich auch in den Anreizsystemen für die Manager niederschlagen (höhere Anteile kurzfristiger Boni für die Erreichung finanzieller Ziele), aber auch in der „Vermarktlichung " in den Führungs- und Entlohnungssystemen insgesamt. Solche Unternehmen internalisieren den Markt stärker; ein Interesse an kurzfristiger Kapitalverwertung wird verstärkt und Möglichkeiten für eine langfristig angelegte, stetige und die Arbeitnehmer und andere Stakeholder einbeziehende Unternehmensentwicklung werden reduziert. 


\section{LITERATUR}

Barth, J./Capri, G./Levine, R. (2013): Bank regulation and supervision in 180 countries from 1999 to 2011, in: Journal of Financial Economic Policy 5 (2) S. $111-219$

Beyer, J. (2003): Deutschland AG a.D.: Deutsche Bank, Allianz und das Verflechtungszentrum des deutschen Kapitalismus, in: Streeck, W./Höpner, M. (Hrsg.): Alle Macht dem Markt? Fallstudien zur Abwicklung der Deutschland AG, Frankfurt a. M./New York, S. 118-146

BlackRock (2009): Company Report 2009, http://thomson.mobular.net/ thomson/7/3110/4248/

Buchter, H. ( 2015): BlackRock. Eine heimliche Weltmacht greift nach unserem Geld, Frankfurt a. M./New York

Čhihák, M./Demirgüç-Kunt, A./Feyen, E./Levine, R. (2012): Benchmarking financial systems around the world: World Bank, Working Paper (6175), Washington, D.C.

Deutsche Bundesbank (2014): Eigentümerstruktur am deutschen Aktienmarkt: allgemeineTendenzen und Veränderungen in der Finanzkrise, Monatsbericht September, S. 19-33

Fligstein, N./Brantley, P. (1992): Bank control, owner control, or organizational dynamics: Who controls the large modern corporation?, in: American Journal of Sociology 98 (2) S. 280-307

Hall, P. A./Soskice, D. (Hrsg.) (2001): Varieties of Capitalism. The Institutional Foundations of Comparative Advantage, Oxford

Harvey, D. (2014): Das Rätsel des Kapitals entschlüsseln, Hamburg

Hein, E. (2005): Finanzstruktur und Wirtschaftswachstum - theoretische und empirische Aspekte: IMK in der Hans-Böckler-Stiftung, IMK Studies (1), Düsseldorf Heires, M./Nölke, A. (2011): Finanzkrise und Finanzialisierung, in: Kessler, 0. (Hrsg.): Die Politische Ökonomie der Weltfinanzkrise, Wiesbaden, S. 37-52

Heires, M./Nölke, A. (Hrsg.) (2014): Politische Ökonomie der Finanzialisierung, Wiesbaden

Herman, E. S. (1981): Corporate control, corporate power, Cambridge Heyes, J./Lewis, P./Clark, I. (2012): Varieties of capitalism, neoliberalism and the economic crisis of 2008-?, in: Industrial Relations Journal 43 (3), S. $222-241$

Kremer, D. (2013): Vermögensverwalter Blackrock. Der schwarze Riese, http://www.faz.net/aktuell/finanzen/fonds-mehr/vermoegensverwalterblackrock-der-schwarze-riese-12057048.html

Lapavitsas, C. (2011): Theorizing financialization, in: Work, Employment \& Society 25 (4), S. $611-626$

Levine, R. (2002): Bank-based or market-based financial systems: Which is better?, in: Journal of Financial Intermediation 11 (4), S. 398-428

Marx, K. (1980): Das Kapital. Kritik der politischen Ökonomie, Band 1, Berlin

Mizruchi, M. S./Domhoff, G.W. (1982): The American corporate network,

1904-1974, Beverly Hills

Murray, G. (2006): Capitalist networks and social power in Australia and New Zealand, Aldershot

Murray, G./Scott, J. (Hrsg.) (2012): Financial elites and transnational business. Who rules the world?, Cheltenham [u. a.]

Peetz, D./Murray, G. (2012): The financialization of global corporate ownership in: G. Murray/J. Scott (Hrsg.): Financial elites and transnational business. Who rules the world?, Cheltenham u. a., S. 26-53

Peetz, D./Murray, G./Nienhüser, W. (2013): The new structuring of corporate ownership, in: Globalizations 10 (5), S. 711-730

Schneider, M. R./Paunescu, M. (2012): Changing varieties of capitalism and revealed comparative advantages from 1990 to 2005: a test of the Hall and Soskice claims, in: Socio-Economic Review 10 (4), S. 731-753

Streeck, W./Höpner, M. (Hrsg.) (2003): Alle Macht dem Markt? Fallstudien zur Abwicklung der Deutschland AG, Frankfurt a. M./New York

Unterreiner, V. (2011): Die Besitzer der Welt, in: Die Welt vom 24.04., http:// www.welt.de/print/wams/wirtschaft/article13252677/Die-Besitzer-der-Welt.html (letzter Zugriff: 22.07.2016)

Van der Zwan, N. (2014): Making sense of financialization, in: Socio-Economic Review 12 (1), S. 99-129

Van Treeck, T. (2009): The political economy debate on 'financialization'- a macroeconomic perspective, in: Review of International Political Economy 16 (5), S. 907-944

Van Treeck, T./Hein, E./Dünhaupt, P. (2007): Finanzsystem und wirtschaftliche Entwicklung in den USA und in Deutschland im Vergleich. Eine makroökonomische Skizze, in: WSI Mitteilungen 60 (12), S. 635-642, http://www.boeckler. de/wsi-mitteilungen_25007_25017.htm
Vitali, S./Glattfelder, J. B./Battiston, S. (2011): The network of global corporate control, in: PloS one 6 (10), S. e25995

Vitols, S. (2004): Changes in Germany's bank-based financial system: a varieties of capitalism perspective: Wissenschaftszentrum Berlin für Sozialforschung, Discussion papers, http://hdl.handle.net/10419/51230

Windolf, P. (1994): Die neuen Eigentümer: Eine Analyse des Marktes für Unternehmenskontrolle, in: Zeitschrift für Soziologie 23 (2), S. 79-92

Windolf, P. (2013): Institutionelle Eigentümer in Finanzmarkt-Kapitalismus, in: B. Emunds/W.-G. Reichert (Hrsg.): Den Geldschleier lüften! Perspektiven auf die monetäre Ordnung in der Krise, Marburg, S. 207-229

Windolf, P./Nollert, M. (2001): Institutionen, Interessen, Netzwerke: Unternehmensverflechtungen im internationalen Vergleich, in: Politische Vierteljahresschrift 42 (1), S. 51-78

Worstall, T. (2011): Six waltons have more wealth than the bottom $30 \%$ of Americans, http://www.forbes.com/sites/timworstall/2011/12/14/six-waltonshave-more-wealth-than-the-bottom-30-of-americans/

\section{AUTOREN}

WERNER NIENHÜSER, Prof. Dr., Professur für Betriebswirtschaftslehre, insbesondere Arbeit, Personal und Organisation, an der Fakultät für Wirtschaftswissenschaften der Universität Duisburg-Essen. Arbeitsschwerpunkte: Arbeitsbeziehungen/Employment Relations, Arbeitnehmer-Mitbestimmung, Macht in und von Organisationen

werner.nienhueser@uni-due.de

DAVID PEETZ, Prof. Dr., Professur für Employment Relations Centre for Work, Organisation and Wellbeing der Griffith University in Brisbane, Australien. Arbeitsschwerpunkte: Beziehungen zwischen Kapital und Arbeit, Tarifverhandlungen, Gewerkschaften.

dpeetz@griffith.edu.au

Georgina Murray, Prof. Dr., Professur für Politische Ökonomie am Centre for Work, Organisation and Wellbeing der Griffith University in Brisbane, Australien. Arbeitsschwerpunkte: Politische Ökonomie, feministische Theorie, globaler Kapitalismus.

g.murray@griffith.edu.au 